\title{
Effect of 11-Mercaptoundecylphosphoric-acid Layer Formation on Gold Surfaces Interacting with Titanium Dioxide Surfaces
}

\author{
Jin-Won Park \\ Department of Chemical Engineering, College of Engineering, Seoul National University of Science and Technology, \\ Seoul 139-743, Korea.E-mail: jwpark@snut.ac.kr \\ Received June 9, 2010, Accepted August 20, 2010
}

\begin{abstract}
We studied effects of the 11-Mercaptoundecylphosphoric-acid layer formation on gold surfaces that have the interactions with the titanium dioxide surface for design of gold- titanium dioxide distribution. The atomic force microscope (AFM) was used to measure forces between the surfaces as a function of the salt concentration and $\mathrm{pH}$ value. The forces were analyzed with the DLVO (Derjaguin-Landau-Verwey-Overbeek) theory, to evaluate the potential and charge density of the surfaces quantitatively for each salt concentration and each $\mathrm{pH}$ value. The interpretation for the evaluation was performed with the law of mass action and the ionizable groups on the surface.
\end{abstract}

Key Words: 11-Mercaptoundecylphosphoric-acid, Au-TiO 2 catalyst, AFM, DLVO theory, Surface forces

\section{Introduction}

Materials composed of gold and a semiconducting support such as titanium dioxide have been considered for numerous applications for example in surface patterning, catalysis, photocatalysis, and photovoltaic cells. ${ }^{1-6}$ For the catalysis, the oxidation on the materials by light generates electron-transfer processes that may be used for the degradation of the organic pollutants. $^{7}$ Activity and selectivity of such materials and catalysts is correlated with the distribution. ${ }^{8}$

Different methodologies were developed in the past for the preparation of $\mathrm{Au}-\mathrm{TiO}_{2}$ catalyst materials such as metal ion impregnation and deposition-precipitation followed by drying, calcinations, and reduction. ${ }^{9}$ During these procedures, the $\mathrm{Au}$ particles or clusters are formed directly on the support. However, these approaches have some drawbacks. The non-uniform distribution of the precursors in the solution, caused by the gravitational forces, leads to various dispersions of the resulting metal particles. The essential thermal treatment induces severe agglomeration problems and may cause undesirable change in the support chemistry through ionic diffusion. ${ }^{5}$ Therefore, alternative approach for catalyst preparation has been suggested. The approach is relevant to the preparation of metallic $\mathrm{Au}^{0}$ particles in solution and subsequent deposition on the $\mathrm{TiO}_{2}{ }^{5,10}$ This approach is based on passivating ligands such as phosphines and thiols, which play a role to prevent the particles from agglomeration in solution. ${ }^{11-13}$ The ligands can be removed by calcinations of the material and thiolate oxidation, after the gold particles are deposited on the $\mathrm{TiO}_{2}{ }^{14-17}$

The atomic force microscope (AFM) was employed to monitor the behavior of gold particles. ${ }^{18}$ Among surface characterization techniques, the AFM is a powerful technique to provide insight for analysis of surface properties of a colloidal particle and a flat surface as a function of separation. ${ }^{19}$ The properties, especially electrostatic properties, were estimated by fitting the force curves, acquired from the AFM, with Derjaguin-LandauVerwey-Overbeek (DLVO) theory. ${ }^{20}$ The surface properties are an indicator for electrostatic repulsive force between parti- cles, which may strongly affect on the particle distribution that determines the activity and selectivity of the catalyst. In this paper, we studied the effects of the 11-Mercaptoundecylphosphoric-acid layer formation on gold surfaces that have the interactions with the titanium dioxide surface.

\section{Experiments}

Surface preparation. The gold surfaces were prepared by using a high-vacuum electron beam evaporator to sequentially deposit a $5 \mathrm{~nm}$ chrome adhesion layer and a $100 \mathrm{~nm}$ gold layer on the silicon wafers. Immediately prior to use, the gold surfaces were cleaned in a $4: 1$ solution of $96 \%$ sulfuric acid and $30 \%$ hydrogen peroxide at $60-80{ }^{\circ} \mathrm{C}$ for $5 \mathrm{~min}$. The gold surface was immersed in a solution of $10 \mathrm{mM} 11$-mercaptoundecylphosphoric-acid, $100 \mathrm{mM}$ potassium nitride, at $\mathrm{pH} 4$ for several hours at room temperature, followed by rinsing with a running buffer. The immobilization of 11-mercaptoundecylphosphoricacid was confirmed from qualitative surface force measurement in $100 \mathrm{mM}$ potassium nitride at $\mathrm{pH} 4$. For quantitative surface force measurements, the solution was replaced with a running buffer (There are six running buffers used in this research 100,10 , and $1 \mathrm{mM}$ potassium nitride at $\mathrm{pH} 4$ and 8 , respectively). $\mathrm{TiO}_{2}$ layer was deposited on the surface of the silicon wafer by sputtering titanium in an argon-oxygen environment for 40 min using an RF Magnetron source operating at $2 \mathrm{~kW}$. Prior to sputtering, the wafers were dipped in hydrofluoric acid to remove any native oxide layer. The total pressure used was $5 \times$ $10^{-6}$ bar, with argon and oxygen flow rates of 6 and $1.2 \mathrm{dm}^{3} / \mathrm{min}$, respectively. The substrate was rotated continuously during sputtering. The target to substrate distance was $7 \mathrm{~cm}$ and target diameter was $20 \mathrm{~cm}$. The characteristics of the $\mathrm{TiO}_{2}$ layers were identical with those of the gold surface.

AFM measurements. Topology images and surface force measurements were made with a 3-D Molecular Force Probe AFM (Asylum Research, Santa Barbara, CA) with a closed-loop piezo-electric transducer. Microfabricated silicon oxynitride cantilevers (Olympus, Shinjuku-ku, Tokyo, Japan) with a nomi- 


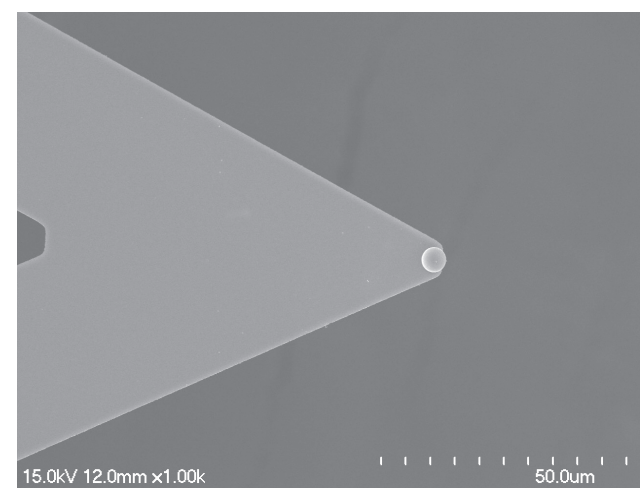

Figure 1. Titanium dioxide sphere-attached-cantilever.

nal radius of $20 \mathrm{~nm}$ were used for topographic imaging and qualitative surface force measurements. Quantitative surface force measurements were made with titanium oxide spheres (Microspheres-Nanospheres, Cold Spring, NY) of $3 \mu \mathrm{M}$ diameter that were attached to the microfabricated cantilevers, shown in Figure 1. The spheres were immobilized at the end of the cantilever with UV-sensitive adhesive (Norland Products, New Brunswick, NJ). Exposure to the UV light source and surface cleaning were achieved in an ozone cleaning apparatus (Jelight, Irvine, CA). It was observed that the exposure did not cause any change in the response of the cantilever. The spring constant of the cantilever was determined from the thermal frequency spectrum of the cantilever. ${ }^{21}$

\section{Theory}

The forces between interacting electrostatic double layers have been described with the theory of Derjaguin-LandauVerwey-Overbeek (DLVO). ${ }^{22}$ According to the theory, the total interaction energy between two plates is the sum of several contributions, including an attractive van der Waals component $\left(V_{A}\right)$ and an electrostatic repulsion or attraction $\left(V_{E}\right)$. It is also observed that the energy includes an additional repulsion $\left(V_{S}\right)$ at close separations resulting from the presence of ordered solvent layers. ${ }^{23-26}$

According to the Derjaguin approximation, the force between a sphere of radius $R_{T}$ and a plate can be related to the energy between plates by the expression ${ }^{27}$

$$
F / R_{T}=2 \pi\left(V_{A}+V_{E}+V_{S}\right)
$$

The van der Waals energy $\left(V_{A}\right)$ in the non-retarded limit is described by an equation of the form

$$
V_{A}=-A_{H} / 12 \pi d^{2}
$$

where $A_{H}$ is the Hamaker constant and $d$ is the separation distance. ${ }^{28}$ The Hamaker constant of the 11-mercaptoundecylphosphoric-acid layer is assumed to be $7.0 \times 10^{-20} \mathrm{~J}$, since the most component of the layer is hydrocarbon. ${ }^{29}$ The Hamaker constant used for the $\mathrm{TiO}_{2}$ surface is $5.0 \times 10^{-20} \mathrm{~J}^{30}$ The electrostatic interaction $\left(V_{E}\right)$ can be expressed by integrating the electrostatic force. $^{31-33}$ Therefore, the interaction for a 1:1 Electrolyte is

$$
V_{E}=-\int_{\infty}^{D}\left\{2 n^{0} k T\left[\cosh \left(\frac{z e \psi}{k T}\right)-1\right]-\frac{\varepsilon}{2}\left(\frac{d \psi}{d z}\right)^{2}\right\} d z
$$

where $\psi$ is the electrostatic potential. The first term in eq 3 is a repulsive osmotic component that results from the accumulation of charge in the gap between the plates, and the second is a Maxwellian stress that represents an induced charge and is always attractive. To determine $V_{E}$ explicitly, the electrostatic potential as a function of distance between the surfaces must be known. This can be accomplished by solving the PoissonBoltzmann equation

$$
\frac{d^{2} \psi}{d z^{2}}=-\frac{1}{\varepsilon_{0} \varepsilon_{r}} \sum_{i} n_{i}^{0} z_{i} e \exp \left(-\frac{z_{i} e \psi}{k T}\right)
$$

In general, the complete nonlinear form of eq 4 must be solved, which can be found only by numerical techniques. ${ }^{34}$ Then, eq 3 was integrated with a Simpsons' $3 / 8$ rule. The additional repulsive force $\left(V_{S}\right)$ in eq 1 is considered to arise from the presence of ordered solvent layers and can be described by a decaying oscillatory force. ${ }^{35}$ This repulsive force is not clearly understood and will be neglected in the calculations presented here.

\section{Results and Discussion}

The AFM was utilized to characterize the structures of the 11mercaptoundecylphosphoric-acid layer formed on the gold surfaces. Constant force AFM images were acquired on the gold surfaces, 11-mercaptoundecylphosphoric-acid-formedgold surfaces, and $\mathrm{TiO}_{2}$ surfaces. The morphologies of these surfaces were dominated by the polycrystalline structure of the evaporated metals with $1.5 \mathrm{~nm}$ roughness. The morphologies of the surfaces were essentially indistinguishable (results were not shown). Phase separation in lipid and fatty acid films has been observed with AFM and is easily visualized as micrometersized domains in the contact imaging mode. ${ }^{36}$ No observation of these domains on the surfaces strongly suggests that the 11mercaptoundecylphosphoric-acid forms homogeneous layer on the gold surfaces.

For the confirmation of the formation of 11-mercaptoundecylphosphoric-acid layer on the gold surfaces, AFM force measurements were conducted on the gold surfaces and the 11mercaptoundecylphosphoric-acid-formed-gold surfaces with 20-nm-radius probes. As shown in Figure 2, approaching forcedistance curves were purely repulsive for measurements between a silicon nitride probe and the 11-mercaptoundecylphosphoric-acid layer formed in $100 \mathrm{mM}$ potassium nitride at $\mathrm{pH} 4$. The observed forces were approximately $0.2 \mathrm{nN}$ less than $2.0 \mathrm{~nm}$, while the force curve of the gold surface showed an negative force that means an attractive region. The repulsion, not found on the gold surfaces, appears to be due to the lower 


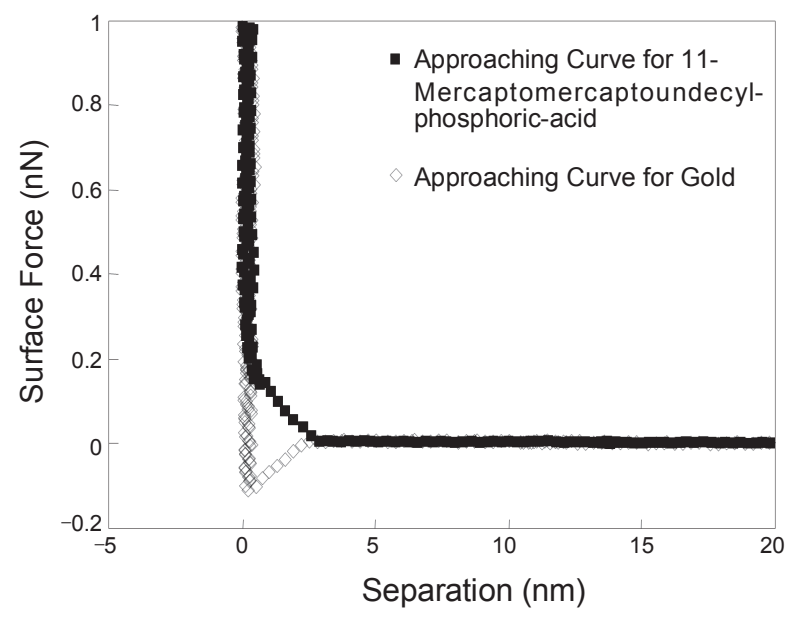

Figure 2. Force-distance curve between a silicon nitride probe and the 11 -mercaptoundecylphosphoric-acid layer formed in $100 \mathrm{mM}$ potassium nitride at $\mathrm{pH} 4$.

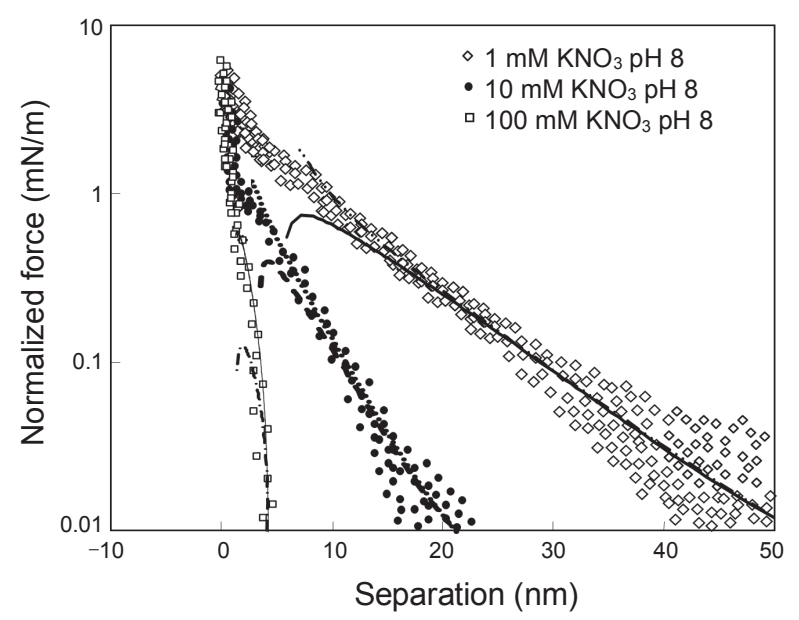

Figure 3. Approaching force curve as a function of the separation between the sphere and the surface of the titanium dioxide in 1, 10, 100 $\mathrm{mM}$ potassium nitride at $\mathrm{pH} 8$.

Hamaker constant and more hydration of the 11-mercaptoundecylphosphoric-acid layer. ${ }^{29}$ The clear difference of the force curve indicates that the 11-mercaptoundecylphosphoric-acid layers were well-formed on the gold surfaces.

The theoretical analysis of the force curves between the titanium dioxide surface and the 11-mercaptoundecylphosphoricacid layer surface requires that the titanium dioxide surface should be analyzed earlier, because the analysis is based on the asymmetric boundary conditions. Therefore, as a first, the surface charge density and potential of the $3-\mu \mathrm{M}$-diameter titanium dioxide sphere were estimated from the analysis of the force curves between the sphere and the titanium dioxide surface. The approaching force curves presented in Figure 3 were acquired as a function of the separation between the titanium dioxide sphere and the titanium dioxide surface in three running buffers at $\mathrm{pH}$ 8. The long-range surface forces were completely repulsive, and their range was highly influenced by the ionic strength of the solution. The exponential dependence of the

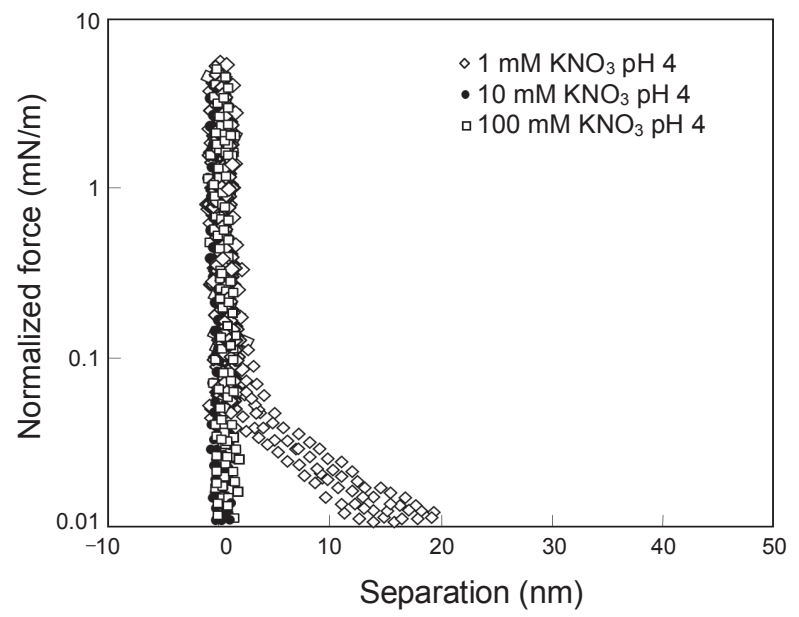

Figure 4. Approaching force curve as a function of the separation between the sphere and the surface of the titanium dioxide in 1,10 , $100 \mathrm{mM}$ potassium nitride at $\mathrm{pH} 4$.

repulsive force on distance was consistent with double-layer forces between surfaces of like charge in aqueous solutions. At separations of below around $2.0 \mathrm{~nm}$, short-range repulsive forces were clearly observed that may be attributed to the steric forces and the inherent roughness of the surfaces. ${ }^{37,38}$

In contrast with $\mathrm{pH} 8$, the long-range surface forces at $\mathrm{pH} 4$ less repulsive for potassium nitrate solutions. The forces were presented in Figure 4 for $\mathrm{pH} 4$. The repulsive forces, observed in $1 \mathrm{mM}$ potassium nitrate solution at $\mathrm{pH} 4$, were smaller than those at $\mathrm{pH} 8$ due to the iso-electric point of $\mathrm{TiO}_{2}$. At pH 4, the forces were not even found in $10 \mathrm{mM}$ and $100 \mathrm{mM}$ potassium nitrate solution. That is, at $\mathrm{pH} 4$, the electrostatic forces in the long range appeared not to be the dominant component of the surface forces on the $\mathrm{TiO}_{2}$ surface in $10 \mathrm{mM}$ and $100 \mathrm{mM}$ potassium nitrate solution. Therefore, in $10 \mathrm{mM}$ and $100 \mathrm{mM}$ potassium nitrate solution at $\mathrm{pH} 4$, the analysis of the DLVO theory was not suitable for the force curves so that the surface potential and charge density could be acquired.

The long-range surface forces were analyzed with the DLVO theory to evaluate constant surface potential or charge density of the surfaces. The results of this theoretical analysis are summarized in Table 1. The surface potential of the titanium dioxide surface is found -10 to $-100 \mathrm{mV}$ at $\mathrm{pH}$ 8.0. Our results seem to be consistent with those of Feiler et al., where AFM was utilized for the characterization of the titanium dioxide surface in a $1 \mathrm{mM}$ potassium nitrate solution and the surface potential was $-43 \mathrm{mV}$ at $\mathrm{pH} 8 .^{30}$ The change from negative to positive potential is caused by the change from negative to positive values at an isoelectric point of $\mathrm{pH} 4.3 .^{30}$ For the $10 \mathrm{mM}$ and $100 \mathrm{mM}$ potassium nitrate solution at $\mathrm{pH} 4$, the surface potential and charge density of the $\mathrm{TiO}_{2}$ were not acquired.

As presented in Table 1, the surface potential of the titanium dioxide surface increased monotonically with decreasing ionic strength, while the surface charge density deceased monotonically with decreasing ionic strength at $\mathrm{pH} 8$. A model has been developed to describe the salt concentration dependence of the surface potential and charge density of a surface with ionizable groups based on the law of mass action. ${ }^{39}$ The relation bet- 
Table 1. Electrostatic properties of the titanium dioxide surfaces

\begin{tabular}{lccc}
\hline \multicolumn{3}{c}{$\mathrm{pH} 8$} \\
\hline & $1 \mathrm{mM}$ Potassium nitrate & $10 \mathrm{mM}$ Potassium nitrate & $100 \mathrm{mM}$ Potassium nitrate \\
\hline Surface potential $(\mathrm{mV})$ & $-43 \pm 3$ & $-26 \pm 3$ & $-17 \pm 2$ \\
Surface charge density $\left(10^{-3} \mathrm{C} / \mathrm{m}^{2}\right)$ & $-3.0 \pm 0.3$ & $-6.6 \pm 0.6$ & $-12.4 \pm 1.3$ \\
\hline & $\mathrm{pH} 4$ & $10 \mathrm{mM}$ Potassium nitrate & 100 mM Potassium nitrate \\
\hline Surface potential $(\mathrm{mV})$ & $1 \mathrm{mM}$ Potassium nitrate & $-^{a}$ & $-^{a}$ \\
Surface charge density $\left(10^{-3} \mathrm{C} / \mathrm{m}^{2}\right)$ & $9 \pm 1$ & $-{ }^{a}$ & ${ }^{a}$ \\
\hline
\end{tabular}

${ }^{a}$ Electrostatic property was not acquired.

ween surface charge density $(\sigma)$, surface potential $\left(\psi_{o}\right)$, and salt concentration may be determined by the simultaneous solution of the law of mass action

$$
\begin{aligned}
& \sigma=\sigma_{o} \\
& \frac{1}{1+K_{a}\left[H^{+}\right] \exp \left(-\frac{e \psi_{o}}{k T}\right)+K_{K^{+}}\left[K^{+}\right] \exp \left(-\frac{e \psi_{o}}{k T}\right)}
\end{aligned}
$$

and Graham's equation

$$
\sigma=\sqrt{8 \varepsilon \varepsilon_{o} k T} \sinh \left(\frac{e \psi_{o}}{k T}\right) \sqrt{\left(\left[K^{+}\right]+\left[H^{+}\right]\right)}
$$

where $\sigma_{o}$ is the maximum surface charge density, $\psi_{o}$ is the surface potential, $\varepsilon$ is the dielectric constant of water, $\varepsilon_{o}$ is the permittivity of free space, $e$ is the electronic charge constant, $k$ is the Boltzmann's constant, $T$ is temperature. The surface potential and charge density dependence on the salt concentration, found from the measurement at $\mathrm{pH} 8.0$, were consistent with the prediction from the model.

After the characterization of the titanium dioxide sphere surface, the force measurements were performed on the 11mercaptoundecylphosphoric-acid layer formed on the gold surfaces with the titanium dioxide sphere. The surface potential and charge density of the 11-mercaptoundecylphosphoric-acid layer surfaces were found by analyzing the force curves. Figure 5 shows the results of force measurements made on the 11mercaptoundecylphosphoric-acid layers in the three running buffers at $\mathrm{pH} 8$. The long-range surface forces were purely repulsive and varied in range with ionic strength in a manner that was consistent with double-layer forces. The surface forces were analyzed quantitatively with the DLVO theory using asymmetric boundary conditions. In Table 2, the surface potentials and charge densities on the 11-mercaptoundecylphosphoric-acid layer surfaces were summarized as a function of ionic strength and $\mathrm{pH}$ value.

The surface potential and charge density of the 11-mercaptoundecylphosphoric-acid layer surface were also described, as a function of the salt concentration, with the model. It was found that the 11-mercaptoundecylphosphoric-acid layer had

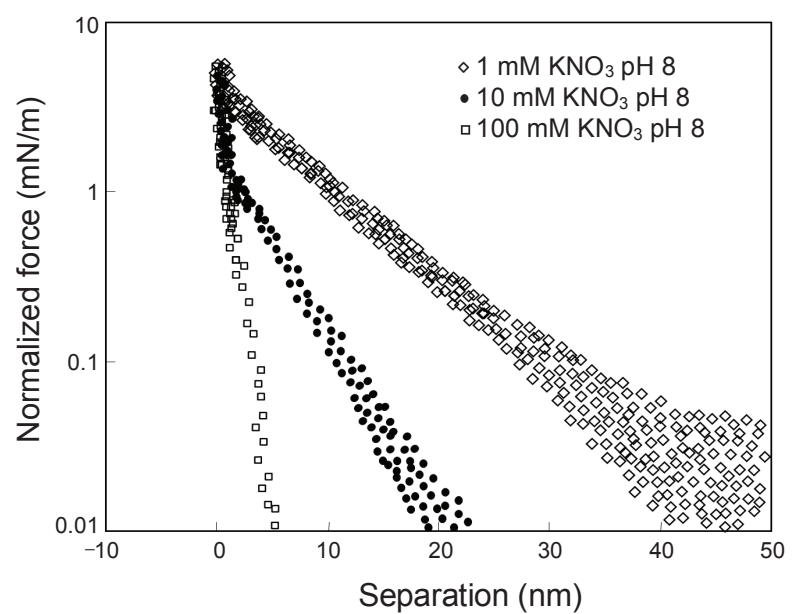

Figure 5. Approaching force curve as a function of the separation between the titanium dioxide sphere and the 11-mercaptoundecylphosphoric-acid layer in $1,10,100 \mathrm{mM}$ potassium nitride at $\mathrm{pH} 8$.

higher values for the surface charge densities and potentials than the titanium dioxide surfaces at $\mathrm{pH} 8$, which may be attributed to the ionized-functional-groups of the 11-mercaptoundecylphosphoric-acid layer. In $1 \mathrm{mM}$ potassium nitrate solution at $\mathrm{pH} 4$, the long-range-forces were purely attractive on the 11mercaptoundecylphosphoric-acid layer surface (Figure 6), while they were repulsive on the titanium dioxide surfaces. This result was predictable due to $\mathrm{p} K_{\mathrm{a}}$ of the 11-mercaptoundecylphosphoric-acid and the iso-electric-point of $\mathrm{TiO}_{2}$. The long-rangeforces on the 11-mercaptoundecylphosphoric-acid layer surface were not found in $10 \mathrm{mM}$ and $100 \mathrm{mM}$ potassium nitrate solution at $\mathrm{pH} 4$, as they were not on the titanium dioxide surfaces either.

The observations described above suggest that the electrostatic forces between the 11-mercaptoundecylphosphoricacid layer surface and $\mathrm{TiO}_{2}$ surfaces were found to be adjustable quantitatively with a salt concentration and $\mathrm{pH}$ value. From this fact, it was believed that the kinetics of the adsorption for either $\mathrm{TiO}_{2}$ particles to 11-mercaptoundecylphosphoric-acid layer-coated-gold surface or 11-mercaptoundecylphosphoricacid layer-coated-gold particles to $\mathrm{TiO}_{2}$ surface might be also adjustable, because the adsorption is determined by the surface forces between the surfaces. Furthermore, the kinetics determines the distribution of the particles adsorbed to the surface. 
Table 2. Electrostatic properties of the 11-mercaptoundecylphosphoric-acid layer

\begin{tabular}{lcrr}
\hline \multicolumn{3}{c}{$\mathrm{pH} 8$} \\
\hline Surface potential $(\mathrm{mV})$ & $1 \mathrm{mM}$ Potassium nitrate & $10 \mathrm{mM}$ Potassium nitrate & 100 mM Potassium nitrate \\
Surface charge density $\left(10^{-3} \mathrm{C}^{2} \mathrm{~m}^{2}\right.$ & $-92 \pm 5$ & $-57 \pm 5$ & $-32 \pm 3$ \\
\hline & $-15 \pm 2$ & $-27 \pm 3$ & $-50 \pm 5$ \\
\hline Surface potential $(\mathrm{mV})$ & $1 \mathrm{mM}$ Potassium nitrate & 10 mM Potassium nitrate & 100 mM Potassium nitrate \\
Surface charge density $\left(10^{-3} \mathrm{C} / \mathrm{m}^{2}\right)$ & $-22 \pm 2$ & $-^{a}$ & $-{ }^{a}$ \\
\hline
\end{tabular}

${ }^{a}$ Electrostatic property was not acquired.

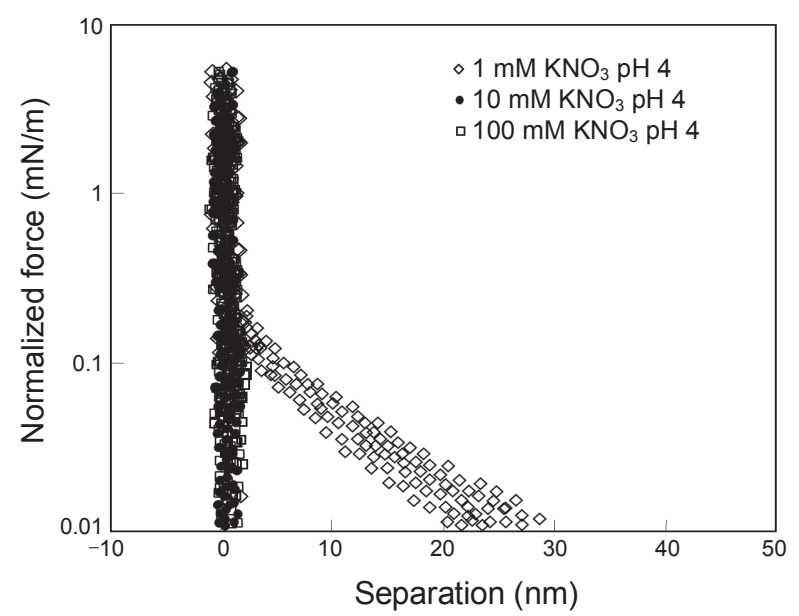

Figure 6. Approaching force curve as a function of the separation between the titanium dioxide sphere and the 11-mercaptoundecylphosphoric-acid layer in 1, 10, $100 \mathrm{mM}$ potassium nitride at $\mathrm{pH} 4$.

Therefore, for the design of the distribution of the particles adsorbed to the surfaces, the surface forces as a function of the salt concentration and the $\mathrm{pH}$ value seem to be important. The distribution may affect on the efficiency of catalysts.

In conclusion, the surface forces between the 11-mercaptoundecylphosphoric-acid layer-coated-gold surface and the $\mathrm{TiO}_{2}$ surface were measured as a function of the salt concentration and $\mathrm{pH}$ value using the AFM. By applying the DLVO theory to the surface forces, the surface potential and charge density of the surfaces were quantitatively estimated as a function of salt concentration and each $\mathrm{pH}$ value. The relation between the salt concentration, and surface potential and charge density was described with the law of mass action, and the $\mathrm{pH}$ dependence was with the ionizable groups on the surface. The electrostatic properties between the 11-mercaptoundecylphosphoric-acid layer-coated-gold surface and the $\mathrm{TiO}_{2}$ surface have not been quantitatively made so far, although the properties are the fundamental information to adjust the distribution of the $\mathrm{TiO}_{2}$ domain on the gold surface or vice versa. This study suggested that the formation of the 11-mercaptoundecylphosphoric-acid layer on gold surfaces may be useful to design the novel structure of either gold particle adsorbed to the $\mathrm{TiO}_{2}$ surface or vice versa by controlling the electrostatic interactions.
Acknowledgments. We thank all of members of Department of Chemical Engineering, the Seoul National University of Science and Technology for help and valuable discussions.

\section{References}

1. Sun, S. Q.; Mendes, P.; Critchley, K.; Diegoli, S.; Hanwell, M.; Evans, S. D.; Leggett, G. J.; Preece, J. A.; Richardson, T. H. Nano Lett. 2006, 6, 345.

2. Peter, A.; Baia, M.; Toderas, F.; Lazar, M.; Tudoran, L. B.; Danciu, V. Studia Universitatis Babes-Bolyai Chemia 2009, 54, 161.

3. Kowalska, E.; Mahaney, O. O. P.; Abe, R.; Ohtani, B. J. Catalys. 2010, 12, 2344.

4. Perlich, J.; Memesa, M.; Diethert, A.; Metwalli, E.; Wang, W.; Roth, S. V.; Timmann, A.; Gutmann, J. S.; Muller-Buschbauma, P. Chem. Phys. Chem. 2009, 10, 799.

5. Li, J.; Zeng, H. C. Chem. Mater. 2006, 18, 4270.

6. Tian, Y.; Tatsuma, T. J. Am. Chem. Soc. 2005, 127, 7632.

7. Kafizasa, A.; Kellicia, S.; Darra, J. A.; Parkin, I. P. J. Photochem. \& Photobiol. A-Chem. 2009, 204, 183.

8. Valden, M.; Lai, X.; Goodman, D. W. Science 1998, 281, 1647.

9. Sakurai, H.; Tsubota, S.; Haruta, M. Applied Catalysis A-General 1995, 102, 125.

10. Li, X.; Fu, J.; Steinhart, M.; Kim, D. H.; Knoll, W. Bull. Korean Chem. Soc. 2007, 28, 1015.

11. Schmid, G. Chem. Rev. 1992, 92, 1709.

12. Jeong, Y.; Han, J. W.; Lee, C.; Noh, J. Bull. Korean Chem. Soc. 2008, 29, 1105.

13. Noh, J.; Park, H.; Jeong, Y.; Kwon, S. Bull. Korean Chem. Soc. 2006, 27, 403.

14. Chou, J.; McFarland, E. W. Chem. Commun. 2004, 14, 1648.

15. Dasog, M.; Scott, R. W. J. Langmuir 2007, 12, 3381.

16. Sandhyarani, N.; Pradeep, T. Chem. Phys. Lett. 2001, 338, 33.

17. Brewer, N. J.; Rawsterne, R. E.; Kothari, S.; Leggett, G. J. J. Am. Chem. Soc. 2001, 123, 4089.

18. Ducker, W. A.; Senden, T. J. Langmuir 1992, 8, 1831.

19. Binnig, G.; Quate, C.; Gerber, G. Phys. Rev. Lett. 1986, 56, 930.

20. Derjaguin, B. V.; Landau, L. Acta Physiochem. 1941, 14, 633.

21. Cleveland, J. P.; Manne, S.; Bocek, D.; Hansma, P. K. Rev. Sci. Instrum. 1993, 64, 403.

22. Derjaguin, B. V. Trans. Faraday Soc. 1940, 36, 203.

23. Israelachvili, J. N.; Adams, G. E. J. Chem. Soc. Faraday Trans. 1978, 74, 975.

24. Shuin, V.; Kekicheff, P. J. Colloid Interface Sci. 1993, 155, 108.

25. Parker, J. L.; Christenson, H. K. J. Chem. Phys. 1988, 88, 8013.

26. O'Shea, S. J.; Welland, M. E.; Pethica, J. B. Chem. Phys. Lett. 1994, 223, 336.

27. Derjaguin, B. V. Kolloid Z 1934, 69, 155.

28. Hartmann, U. Phys. Rev. B 1991, 43, 2404. 
29. Israelachivili, J. N. Intermolecular \& Surface Forces; Academic Press: New York, 1991; pp 183-188, 275-282.

30. Feiler, A.; Jenkins, P.; Ralston, J. Phys. Chem. Chem. Phys. 2000, 2, 5678 .

31. Verwey, E. J. W.; Overbeek, J. T. G. Theory of the Stability of Lyophobic Colloids; Elsevier: New York, 1948; pp 51-63.

32. Hogg, R.; Healy, T. W.; Fuerstenau, D. W. Trans. Faraday Soc. 1966, 62, 1638.

33. Hunter, R. J. Foundations of Colloid Science; Oxford University
Press: Oxford, U.K., 1987; pp 397-409.

34. Chan, D. Y. C.; Pashley, R. M.; White, L. R. J. Colloild Interface Sci. 1980, 77, 283.

35. Parker, J. L. Surf. Sci. 1994, 3, 205.

36. Park, J.-W.; Ahn, D. J. Colloids \& Surf. B: Biointerf. 2008, 62, 157.

37. Ducker, W. A; Senden, T. J.; Pashley, R. M. Nature 1991, 353, 239.

38. Horn, R. G.; Smith, D. T.; Haller, W. Chem. Phys. Lett. 1989, 162, 404.

39. Pashley, R. M. J. Colloid Interface Sci. 1981, 83, 531. 